\title{
Capacitive strain gauges on flexible polymer substrates for wireless, intelligent systems
}

\author{
R. Zeiser ${ }^{1}$, T. Fellner ${ }^{1, *}$, and J. Wilde ${ }^{1}$ \\ ${ }^{1}$ Department for Microsystems Engineering, University of Freiburg, Freiburg, Germany \\ *now at: Robert Bosch GmbH, Reutlingen, Germany \\ Correspondence to: R. Zeiser (roderich.zeiser@imtek.uni-freiburg.de)
}

Received: 13 August 2013 - Revised: 6 March 2014 - Accepted: 15 March 2014 - Published: 10 April 2014

\begin{abstract}
This paper presents a novel capacitive strain gauge with interdigital electrodes, which was processed on polyimide and LCP (liquid crystal polymer) foil substrates. The metallization is deposited and patterned using thin-film technology with structure sizes down to $15 \mu \mathrm{m}$. We determined linear strain sensitivities for our sensor configuration and identified the most influencing parameters on the output signal by means of an analytical approach. Finite-element method (FEM) simulations of the strain gauge indicated the complex interaction of mechanical strains within the sensitive structure and their effect on the capacitance. The influence of geometry and material parameters on the strain sensitivity was investigated and optimized. We implemented thin films on $50 \mu \mathrm{m}$ thick standard polymer foils by means of a temporary bonding process of the foils on carrier wafers. The characterization of the strain sensors after fabrication revealed the gauge factor as well as the cross sensitivities on temperatures up to $100^{\circ} \mathrm{C}$ and relative humidity up to $100 \%$. The gauge factor of a sensor with an electrode width of $45 \mu \mathrm{m}$ and a clearance of $15 \mu \mathrm{m}$ was -1.38 at a capacitance of $48 \mathrm{pF}$. Furthermore, we achieved a substantial reduction of the cross sensitivity against humidity from 1435 to $55 \mathrm{ppm} \%^{-1} \mathrm{RH}$ when LCP was used for the sensor substrate and the encapsulation instead of polyimide. The gauge factor of a sensor half-bridge consisting of two orthogonal capacitors was 2.3 and the cross sensitivity on temperature was reduced to $240 \mathrm{ppm} \mathrm{K}^{-1}$. Finally, a sensor system was presented that utilizes a special instrumentation Integrated Circuit (IC). For this system, performance data comprising cross sensitivities and power consumption are given.
\end{abstract}

\section{Introduction}

Wireless sensor networks are presently under investigation for the condition monitoring of moving machine parts in industrial environments or in mobile systems. The sensor nodes must be suited to measure, store and transmit information about the state of the structure they are mounted on. Typical data of interest are temperature, pressure, acceleration and strain in the surface of the part. Sensor elements applied in such networks should measure the required data with high resolution, often with high $(>1 \mathrm{kHz})$ frequency and low power consumption $(<5 \mathrm{~mW})$. A wired interconnection for power supply and data acquisition is not suitable for sensors on moving parts such as rotating shafts. Additionally, the supply of the sensor systems has to be independent from the main power net of a drive system to guarantee that data will be collected even during a power "black out" for a limited period of time. The strain in the surface of a machine element is an important parameter for condition monitoring. On the basis of strains it is possible to calculate stresses and to detect possible overload of the machine. In stationary applications, resistive strain gauges are usually used for strain measurement. These transducers are supplied and read out with wires. Conventional resistive strain gauges have disadvantages in wireless applications due to their low impedance and therefore high power consumption.

Capacitive sensors are a promising alternative for resistive gauges in the field of wireless strain measurement. Available modern instrumentation ICs can be used to measure 


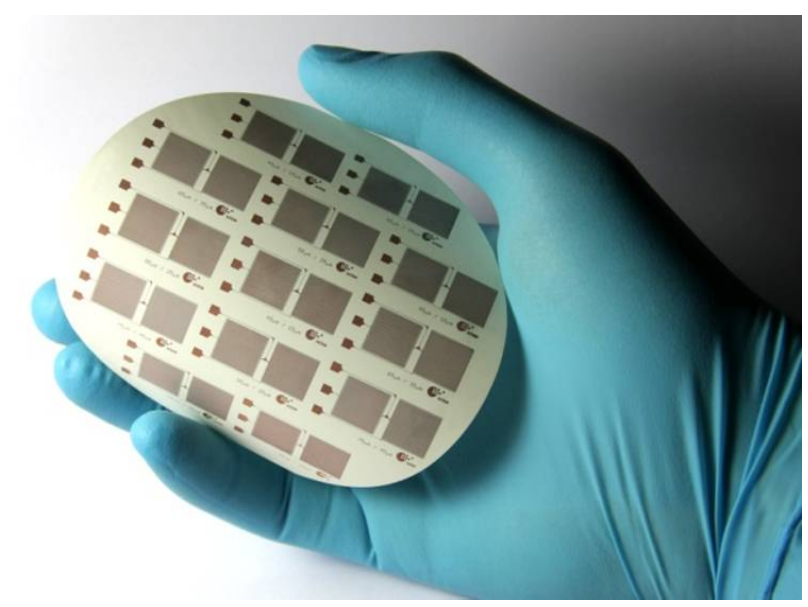

Figure 1. Capacitive strain gauges on LCP foil substrate, directly after processing, and detachment from a carrier wafer.

capacitances with high resolution, at high frequencies with a minimum of power consumption.

In the literature, several concepts for capacitive strain sensors were presented recently (Matsuzaki et al., 2007; Aebersold et al., 2006; Arshak et al., 2000). Our approach combines sensor structures processed with thin-film technology and flexible foil substrates with integrated instrumentation ICs. The capacitive sensor of this study is based on interdigital electrodes with structure heights of about $300 \mathrm{~nm}$, fabricated on flexible polymer foils serving as sensor substrates. Figure 1 depicts capacitive sensor elements on a liquid crystal polymer (LCP) foil after thin-film processing. In this paper we will analyze the substrate material aspects, present the fabrication process and reveal results for both strain sensitivity and cross sensitivities of our strain gauges.

\section{Theory}

In this chapter an analytical model for the sensitivity of the presented strain gauges is given. The sensor principle is an interdigital capacitor with electrodes in a single plane, as exhibited in Fig. 2. The transfer of strain into the sensor structure leads to a change in the clearances of the interdigital electrodes. For an arrangement of the electrodes perpendicular to the strain in the test specimen, the capacitance decreases compared to the status without tensile strain. Igreja et al. (2004) gave an approximation for the computation of the capacitance for interdigital electrodes with Eq. (1) (Igreja et al., 2004). We computed capacitances and strain sensitivities for different interdigital configurations with this analytical formula. Mamishev et al. (2004) presented another method of capacitance computation based on complex transformation (Mamishev et al., 2004.).

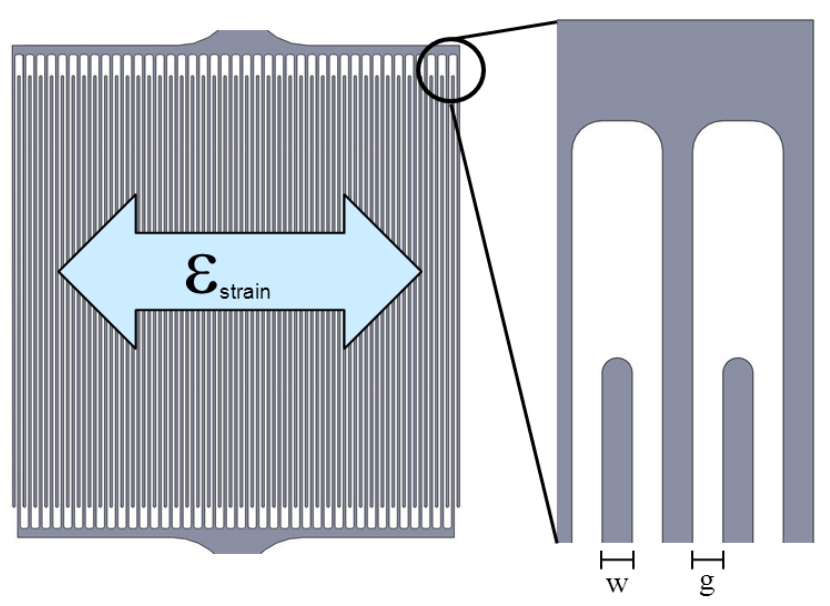

Figure 2. Schematic view of the sensor configuration with interdigital electrodes. Left: working principle of strain detection. Right: geometric electrode parameters - width $w$ and distance $g$.

The capacitance $C$ of two interdigital electrodes can be obtained via following equation:

$C=\varepsilon_{0} \cdot \varepsilon_{\mathrm{r}} \cdot L \cdot \frac{K(k)}{K\left(k^{\prime}\right)}$.

$K(k)$ is the elliptic integral of the first type with the modulus $k$ and the complementary modulus $k^{\prime} . h$ is the electrode height, $g$ is the distance between two electrodes, $w$ is the electrode width and $L$ is the active electrode length. $\varepsilon_{\mathrm{r}}$ represents the material-dependent relative permittivity and $\varepsilon_{o}$ the dielectric constant of free space.

The modulus of $K(k)$ is

$k=\sin \left(\frac{\pi}{2} \eta\right)$

and the complementary modulus

$k^{\prime}=\sqrt{1-k^{2}}$.

Here $\eta$ stands for the geometric relation of electrode width to the electrode pitch,

$\eta=\frac{w}{w+g}$.

Different geometrical configurations of a sensor element have been calculated. The obtained result for a sensor arrangement with electrode width $w=45 \mu \mathrm{m}$, height $h=300 \mathrm{~nm}$ and distance $g=15 \mu \mathrm{m}$ is presented in Fig. 3 . In the following, these geometrical arrangements will be denoted as PI-45/15 and LCP-45/15.

The influence of strain on the electrode distance $g$ is given as follows:

$\varepsilon_{\text {mech }}=\frac{\Delta g}{g}$. 


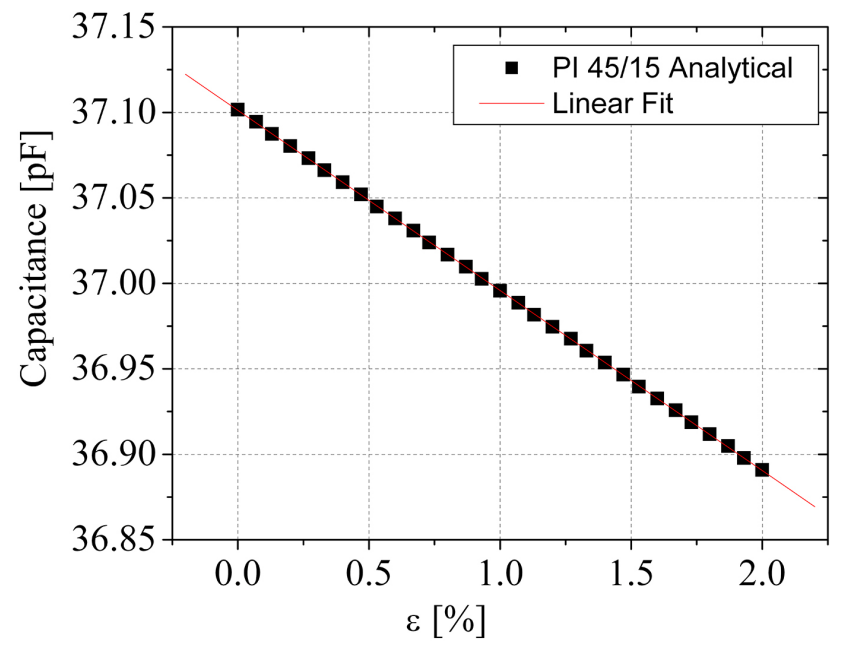

Figure 3. Results for the analytical evaluation of the sensor capacitance of a PI 45/15 as a function of strain.

Equation (6) computes the total capacitance of the interdigital configuration,

$C_{\text {total }}=(N-3) \frac{C_{\mathrm{I}}}{2}+2 \cdot \frac{C_{\mathrm{I}} C_{\mathrm{E}}}{C_{\mathrm{I}}+C_{\mathrm{E}}}$, for $N>3$,

where $N$ is the number of electrode fingers; $C_{\mathrm{I}}$ is the capacitance of two inner electrodes, calculated the same as $C$ in Eq. (1); and $C_{\mathrm{E}}$ stands for exterior electrodes and is computed with the modulus $k_{\mathrm{E}}$ of the elliptic integral $K\left(k_{\mathrm{E}}\right)$ as follows:

$k_{\mathrm{E}}=\frac{2 \sqrt{\eta}}{1+\eta}$.

Figure 3 depicts the results for the capacitance of the interdigital configuration as a function of strain, calculated with the analytical approach. For low strain values, the change in the capacitance is linearly dependent on the change in the electrode distance.

The capacitance of this electrode configuration is $37.1 \mathrm{pF}$.

The gauge factor $G_{\mathrm{F}}$ is one of the principal specification values for strain gauges. For capacitive sensors the gauge factor can be determined as follows:

$G_{\mathrm{F}}=\frac{\Delta C}{C_{0} \cdot \varepsilon_{\text {mech }}}$.

For the presented configuration PI $45 / 15$, the computed gauge factor is -0.3 .

\subsection{FEM simulation of capacitance and strain sensitivity}

In the previous section we verified the linear dependency of strain for the interdigital strain gauge. For investigation of the complex interaction of mechanical strains in a threedimensional material compound and their effect on the capacitance, the analytical approach is unsatisfactory.

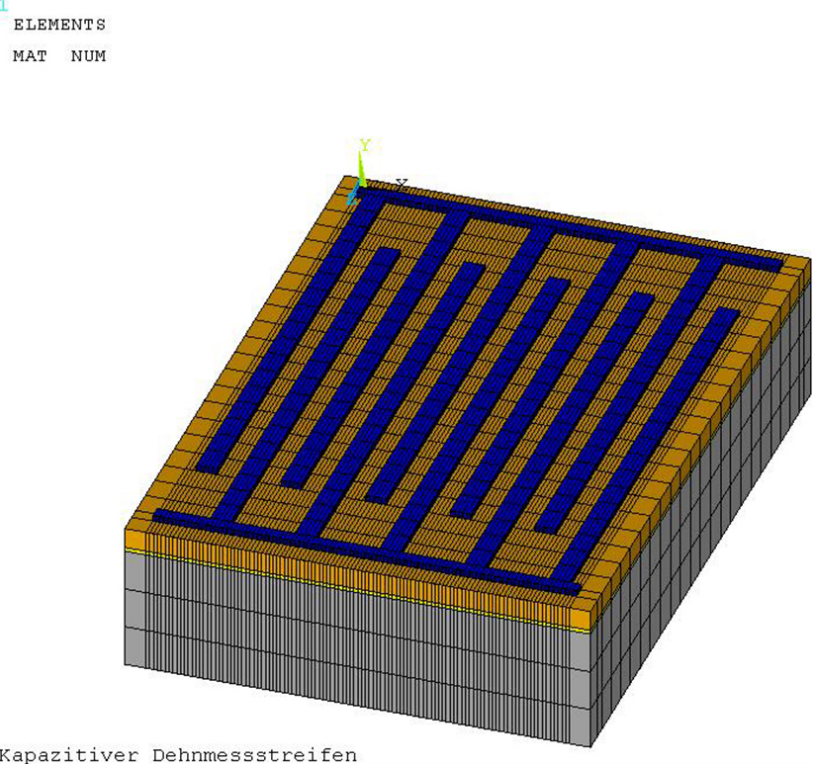

Kapazitiver Dehnmessstreifen

Figure 4. Meshed 3-D FEM model of the sensor structure without encapsulation.

The finite-elements method (FEM) was chosen for the numerical calculation of the sensor capacitance and strain sensitivity. We performed coupled mechanical and electrostatic simulations with the finite-element-simulation software ANSYS. Figure 4 exhibits the meshed 3-D FEM model of the sensor electrodes on a foil substrate, mounted on a test specimen with an adhesive layer. The picture illustrates the geometric and material-dependent arrangement of the brick elements. In the mechanical part of the simulation, different strains in the specimen were defined as the mechanical loads of the system.

The FEM model consists of three-dimensional, eight-node elements of the type SOLID185. After the mechanical simulation, the resulting deformed elements were transformed into electrostatic elements of type SOLID69. The materials parameters, utilzied for the simulations, are presented in Table 1 .

The FEM software computes the capacitance of the strain gauge on the basis of the distribution of the electrostatic field in the dielectric material for a given voltage. Figure 5 shows the contour plot of results for an electrostatic simulation of the finger structures. The concentration of the electrostatic field between the electrodes is illustrated in this figure. One can deduce from this illustration that the effect of capacitance change due to strain is maximized in areas where the maximum electric field is located.

The strain dependency of the sensor output signal turned out to be linear, as predicted by the analytical approach for our sensor configuration. Figure 6 exhibits the results of a simulation for a strain gauge with an electrode width of $45 \mu \mathrm{m}$ and a distance of $15 \mu \mathrm{m}$. 


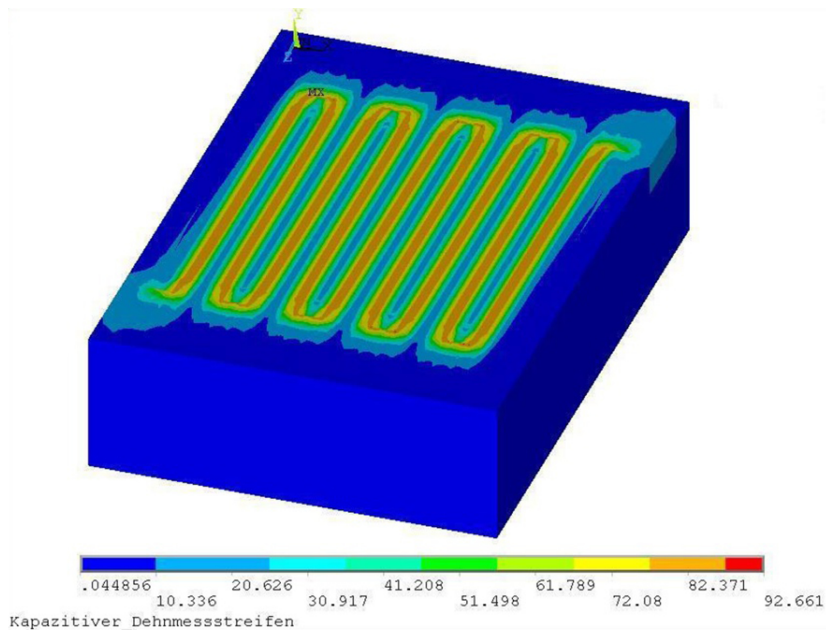

Figure 5. Distribution of the electrostatic field [in $\mathrm{V} \mathrm{m}^{-1}$ ] in the dielectric material for a voltage difference of $5 \mathrm{~V}$ at the interdigital electrodes.

When we varied different design parameters, we found a maximum strain sensitivity of $-0.566 \mathrm{pF} \%^{-1}$ within the fabrication limits for our sensor. A capacitance of $46 \mathrm{pF}$ was obtained for a sensor area of $1 \mathrm{~cm}^{2}$. The corresponding gauge factor $G_{\mathrm{F}}$ of this strain gauge is -1.23 .

Due to a certain strain concentration between the electrodes and a simultaneous decrease in the substrate and encapsulation layer thickness in the loaded case, the gauge factor of the simulation was 4 times higher than when predicted with the analytical approach in the previous section.

We analyzed the influence of geometry and materialdependent parameters of the strain gauge and optimized the system with regard to high strain sensitivity. The optimum structure for a sensor area of $1 \mathrm{~cm}^{2}$ within our process limitations was an electrode of width $w=45 \mu \mathrm{m}$, height $h=300 \mathrm{~nm}$ and distance $g=15 \mu \mathrm{m}$.

Several basic design rules for the sensor layout could be established on the basis of the FEM simulations:

- The sensor substrate should not be thicker than the pitch of the electrodes, because the sensitivity correlates inversely directly with the substrate thickness.

- The gauge factor increases with smaller interdigital distances of the electrodes. For a sensor area of $1 \mathrm{~cm}^{2}$, the sensor structures should be in the range of 10 to $50 \mu \mathrm{m}$.

- As the the thickness of the electrode metallization increases, the characteristic curve of capacitance against strain changes from linear to a hyperbolic behavior.

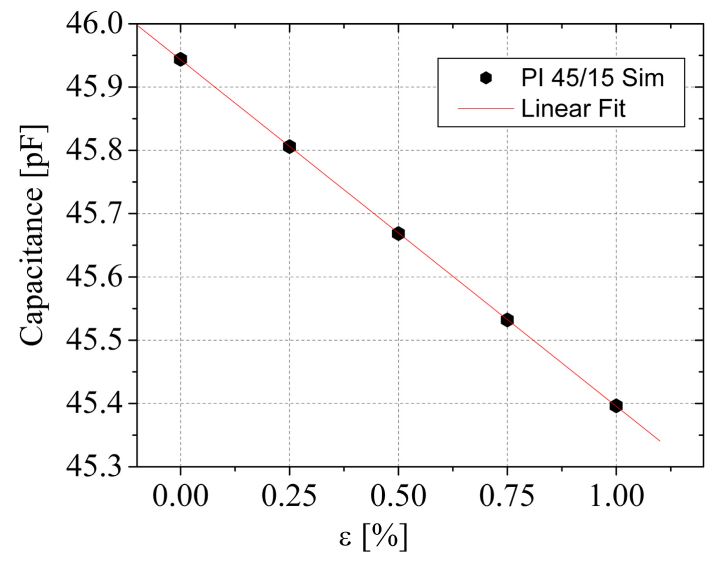

Figure 6. Results for the mechanical-electrostatic FEM simulation of a PI 45/15 sensor capacitance as a function of strain.

\section{Fabrication}

\subsection{Flexible polymer substrate materials}

The choice of the substrate material is crucial for the fabrication of a capacitive sensor. The mechanical and dielectric properties will affect the sensor output characteristics significantly. The principal influences on the capacitance are due to the dielectric properties. Polymer materials are well-suited substrates because a maximum strain transfer can be accomplished due to their low Young modulus and their low back stress on the structure. Hence, the strain is transmitted into the sensor structure with low gauge losses.

The principal foil-based substrate material used in our evaluation is polyimide, which is a standard substrate material used for both resistive strain gauges and flexible PCBs (printed circuit boards). Thin foils of polyimide down to $10 \mu \mathrm{m}$ are available with good thickness conformity and high mechanical strength. Other outstanding material properties are its high thermal and chemical stability. Due to the polymerization route, a high absorption of moisture of approximately $3 \%$ is one disadvantage of polyimide as a sensor material (Melcher et al., 1989).

The second foil material utilized in this study is LCP. This material is mostly known as a material for premolded packages for MEMS sensors. LCP is also applied in the field of high-frequency electronics, mainly because of its low dielectric loss (Zhang, 2006). The value for moisture absorption of LCP is about $0.04 \%$, and approximately 75 times lower than for polyimide (Rogers Corporation, 2008).

\subsection{Process flow}

To obtain the structures with the necessary low electrode pitches, it is necessary to use photolithography for the processing. In this work a lift-off process was chosen for patterning because of its outstanding properties such as accuracy and reproducibility. Structures in the micrometer range 


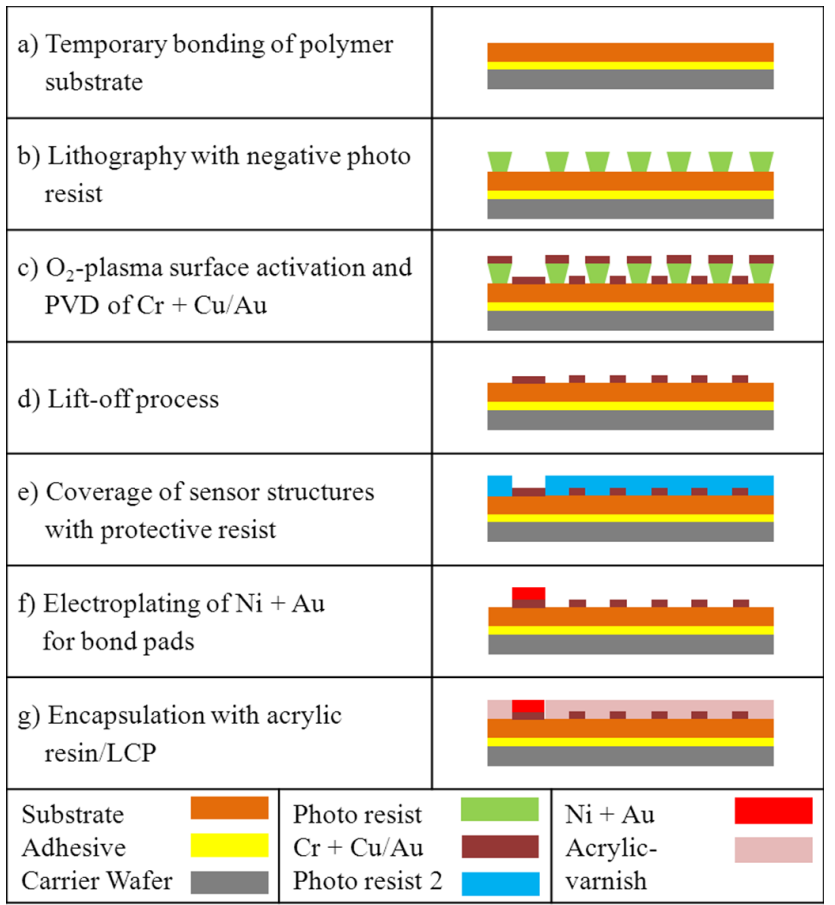

Figure 7. Process flow for the fabrication of capacitive strain gauges.

are possible with this process. Unfortunately, a basic requirement for photolithography is a very flat and rigid substrate. Therefore, a method for processing polymer foils with the approved thin-film technologies in IMTEK's clean room had to be developed in this work. To this end, the foils are temporarily bonded onto carrier substrates with an adhesive that can be removed residue-free after processing is complete.

Figure 7 exhibits a schematic sequence of the sensor structures at every fabrication step, with a brief description.

For the sensor substrates a polyimide foil of type Kapton HN from DuPont and a LCP foil of type Ultralam 3000 from Rogers were used. Step (a) is the temporary bonding of a $50 \mu \mathrm{m}$ polymer foil on a standard silicon wafer, used as a carrier. In this step the carrier wafer is spin-coated with a two-component adhesive and soft-baked at $130^{\circ} \mathrm{C}$. In Fig. 8 temporarily bonded foils of LCP and polyimide are exhibited.

Step (b) comprises spin coating and structuring by photolithography of a negative photoresist from Micro Resist Technology, type ma-N 1420 on the polymer foil. In step (c), an $\mathrm{O}_{2}$ plasma is applied for surface activation and cleaning and subsequently $30 \mathrm{~nm} \mathrm{Cr}$ and $350 \mathrm{~nm} \mathrm{Cu}$ or Au are deposited by the PVD process sputtering. Subsequently the electrodes are patterned by lift-off in step (d). In (e) the sensor electrodes are protected with a photoresist, the interconnection pads are uncovered for an electroplating process in step (f). Thicker films of Ni plus Au were electrodeposited locally to achieve a stable and solderable metallization fin-
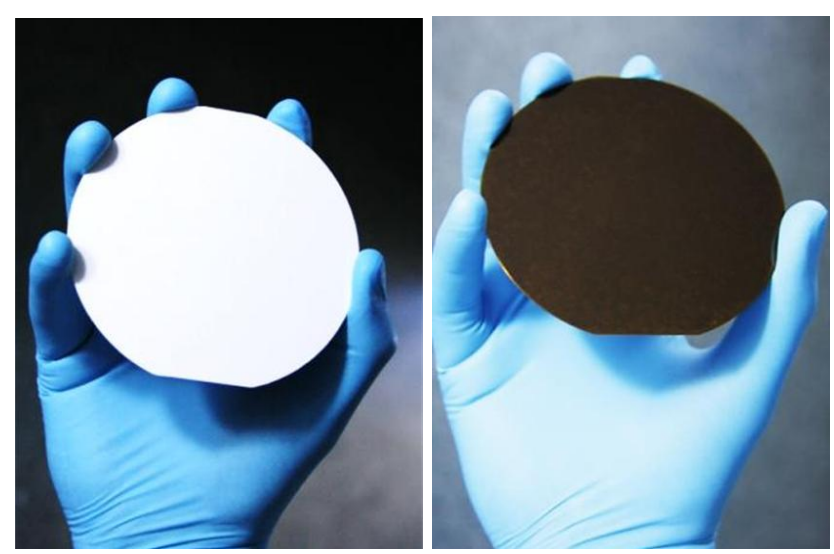

Figure 8. LCP- (left) and polyimide- (right) foils temporarily bonded onto silicon carrier wafers.

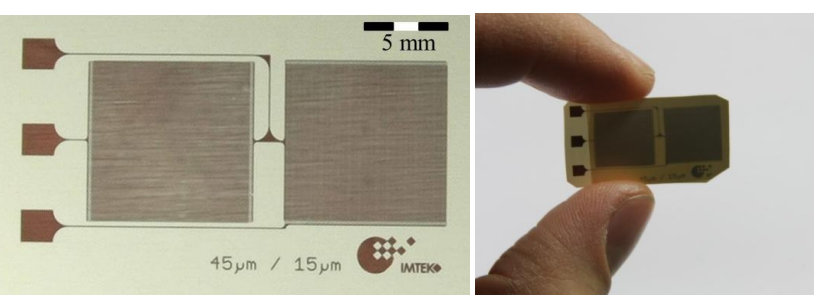

Figure 9. Sensor half bridge (design 1) on LCP substrate (left) and with a protective encapsulation layer of LCP (right).

ish. The sensor structure on polyimide was encapsulated with an acrylic varnish. This varnish was spin-coated on the foil substrate for protection against humidity and particles in step (g).

Due to a low amount of curing agent in the glue, after processing, the foil substrate can be detached from the carrier by heating the assembly up to $20^{\circ} \mathrm{C}$ above the soft-bake temperature without damage. Figure 1 depicts a removed LCP foil carrying sensor structures. Finally, individual sensors are separated from the sheet.

Different variations of the capacitive strain gauges were designed, fabricated and tested in this work. Three design levels were realized: designs 1,2 and 3 . In the first phase, single capacitors and half-bridges of design 1 were investigated for their sensitivity against strain, temperature and humidity. Geometry parameters such as electrode width, length and distance were varied and the measured capacitances were compared to the values calculated analytically and numerically.

Most of the sensors on polyimide and LCP substrate were coated with an acrylic resin of type Plastik 70 from CRC. Also, a transducer type that is fully encapsulated in LCP was manufactured by laminating an additional LCP sheet to the sensor. Both sensor types on LCP are depicted in Fig. 9.

In the second evaluation phase, half-bridges of the sensors design 1 and 2 were bonded directly on a test substrate. As 


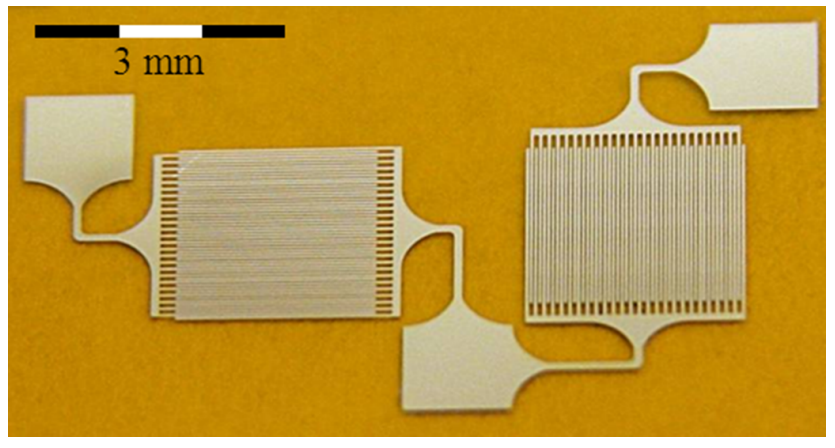

Figure 10. Sensor half-bridge (design 2) on polyimide substrate without encapsulation.

Table 1. Materials parameters applied in the FEM simulation (DuPont, 2008; Rogers Corporation, 2009).

\begin{tabular}{lrrr}
\hline Material & $\begin{array}{r}\text { E modulus } \\
\text { [GPa] }\end{array}$ & $\begin{array}{r}\text { Poission's } \\
\text { ratio }\end{array}$ & $\begin{array}{r}\text { Dielectric } \\
\text { constant }\end{array}$ \\
\hline $\begin{array}{l}\text { Substrate: } \\
\text { polyimide }\end{array}$ & 2.5 & 0.34 & 3.4 \\
$\begin{array}{l}\text { Substrate: } \\
\text { LCP }\end{array}$ & 2.25 & 0.32 & 2.9 \\
$\begin{array}{l}\text { Specimen: } \\
\text { steel }\end{array}$ & 220 & 0.3 & - \\
\hline
\end{tabular}

the two sensor structures are arranged perpendicularly, under the effect of strain, tension will be induced in one structure and compression by transverse contraction in the other. The differential measurement of the capacitances can increase the strain sensitivity and compensate for cross sensitivities. If both capacitances change equally due to temperature, then no change in the difference is detected. Figure 10 depicts an example of a half-bridge configuration for design 2.

In the third phase of this study, we designed a half-bridge sensor layout referred to as design 3 , for the integration of an instrumentation IC directly on the sensor substrate, as shown in Fig. 11.

\section{Experimental}

\subsection{Set-up for strain- and cross-sensitivity measurement}

The experimental setup for the characterization of the strain gauges is presented in this section. A ribbon made of highstrength spring steel with an ultimate elongation of $1 \%$ was designed and fabricated to serve as a test specimen. For the characterization, the sensor prototypes were mounted by means of an acrylic adhesive. Subsequently the ribbon was clamped into a Zwick Z010 universal testing machine with a maximum force capacity of $10 \mathrm{kN}$ in order to generate different levels of strain in the microrange in the steel ribbon.

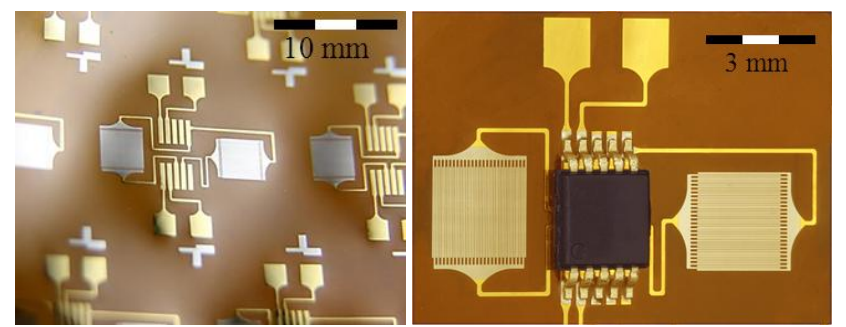

Figure 11. Design 3: capacitive strain sensors (design 3) on polyimide with solderable Ni/Au pads (left), strain gauge with a reflowsoldered $10 \mathrm{I} / \mathrm{O}$ instrumentation IC (right).
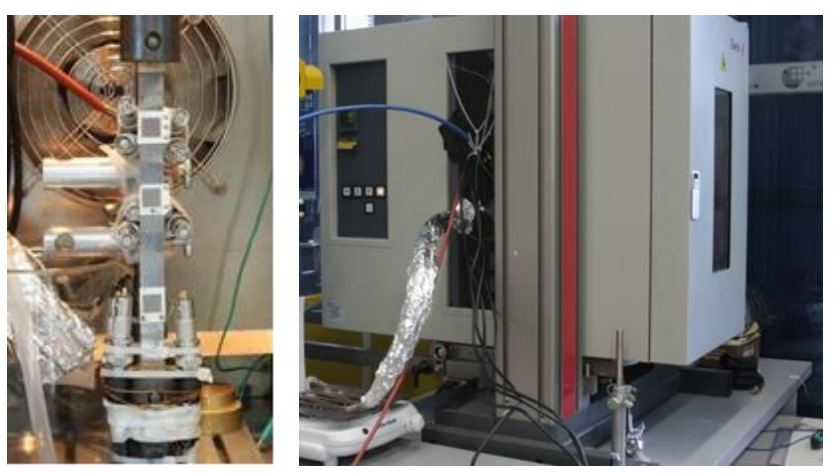

Figure 12. Left: sensors with LCP substrate mounted on a steel specimen; right: heating chamber with lead-through for water vapor, nitrogen and a humidity sensors to generate a controlled $(T, \mathrm{RH})$ environment.

The tensile testing setup is integrated into a heating chamber so that it is possible to measure the influence of temperature on the capacitance and on the gauge factor of the sensor in situ. Figure 12 exhibits three single prototype sensors mounted on the steel ribbon inside this chamber.

A Digimess 300 LCR meter with a resolution of $0.001 \mathrm{pF}$ was used to measure the capacitances in four-wire mode. The signal conductors lead to the outside with coaxial cables in order to shield them from electromagnetic influences. The characterization of the cross sensitivities of the capacitive sensors was a major part of this study. A mixture of nitrogen and water vapor was led into the heating chamber to generate well-defined levels of humidity. For an atmosphere with $0 \%$ relative humidity, only nitrogen gas was used. Temperature and moisture sensors were installed in the heating chamber to control the climate with a precision of approximately $1 \%$. The accuracy of temperature control on the strain gauges was approximately $\pm 2 \mathrm{~K}$.

\subsection{Sensor bridge characterization with an instrumentation IC}

We found high sensitivities for single sensor elements on temperature and humidity. It is possible to compensate for most of the cross sensitivities with an arrangement as a 


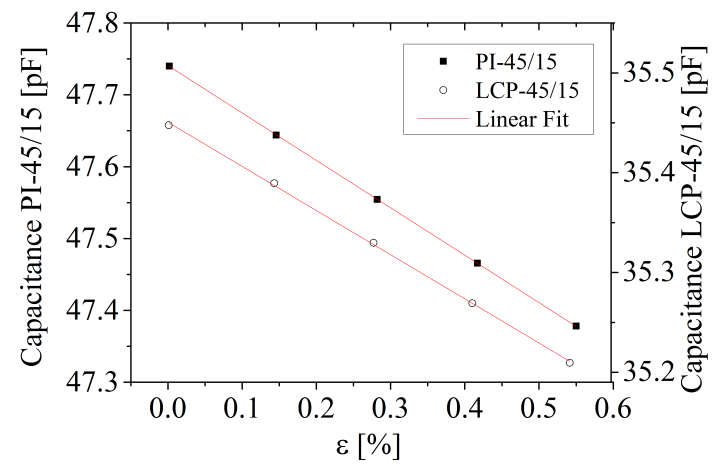

Figure 13. Measured capacitance of single sensors (design 1), PI$45 / 15$ and LCP-45/15 as a function of strain at room temperature in a dry atmosphere.

half-bridge of two capacitive structures on one substrate. When an instrumentation IC is placed close to the capacitive structure, short signal paths are provided and parasitic capacitances are kept low. A low-power AD7152 from Analog Devices with a resolution of 1 bit or $0.25 \mathrm{fF}$ and with a reported power consumption of $0.2 \mathrm{~mW}$ was used for the measurements. Figure 13 shows the strain gauge substrate with an integrated read-out IC. The interdigital sensors of design 3 were in a $45 / 15$ configuration on polyimide, and their sensing area was reduced to $3 \times 3 \mathrm{~mm}^{2}$ to obtain $5 \mathrm{pF}$ per capacitance.

\section{Results and discussion}

\subsection{Strain sensitivity}

The force applied by the tensile testing machine generates a constant strain in the steel ribbon. The analyzed strain steps were $0.145,0.282,0.416$ and $0.545 \%$ for characterization of the strain sensitivity. Figure 13 exhibits the results for a capacitance measurement of a sensor on polyimide with an electrode width of $45 \mu \mathrm{m}$ and a distance of $15 \mu \mathrm{m}$. The temperature during the measurement was $23^{\circ} \mathrm{C}$ and the relative humidity $0 \%$. The slope of the regression line for the measured values is the strain sensitivity of the gauge with $-0.658 \mathrm{pF} \%^{-1}$. The strain coefficient is $S_{\mathrm{C}}=13783 \mathrm{ppm}^{-1}$ when normalized to the absolute capacitance of $C_{0}=47.74 \mathrm{pF}$. The corresponding gauge factor is -1.38 . The capacitance output signal of the gauge is linear over strain, as predicted with the analytical approach and the FEM simulations. Generally, values for the investigated capacitances obtained by FEM correspond to the measured values with a divergence of $4 \%$.

Figure 13 additionally depicts the results for a LCP 45/15 sensor with acrylic varnish encapsulation. The strain coefficient is $S_{\mathrm{C}}=12660 \mathrm{ppm} \%^{-1}$, normalized to the absolute capacitance of $C_{0}=35.45 \mathrm{pF}$. The corresponding gauge factor is -1.27 . The results for both sensor configurations are summarized in Table 2.

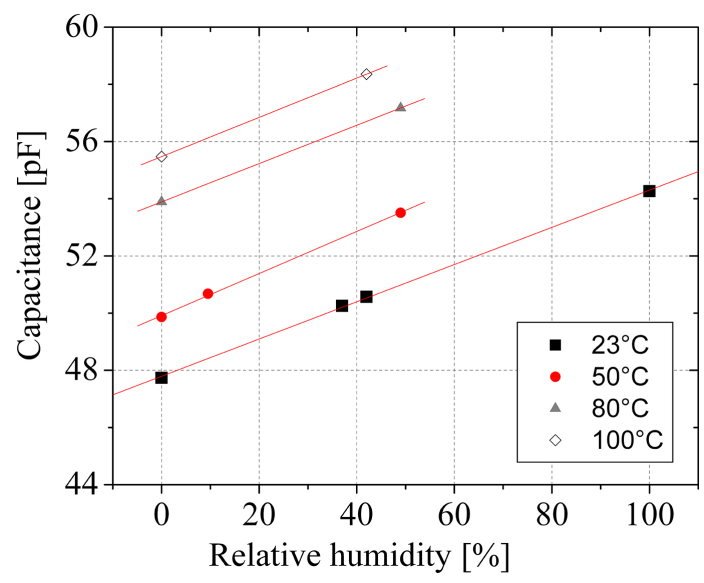

Figure 14. Measured capacitance of a single PI-45/15 sensor (design 1) as a function of relative humidity at different temperatures.

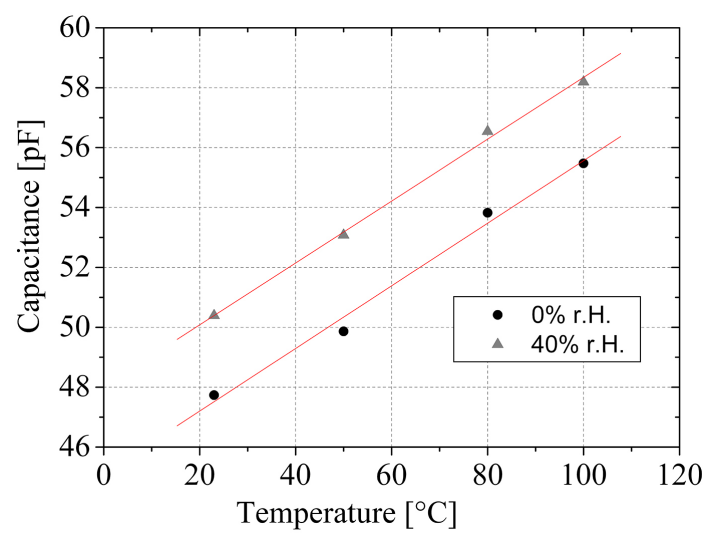

Figure 15. Capacitance of a single PI-45/15 sensor (design 1) as a function of temperature at 0 and $40 \%$ relative humidity.

The capacitance and the gauge factor of the test sensors were obtained at different temperature and humidity levels with the described measuring setup. Figure 14 depicts the results for the capacitance of a strain gauge with polyimide substrate over the test matrix.

The sensor's capacitance shows a linear dependency on temperature at two different values of humidity (Fig. 15). The obtained coefficients for the sensitivities are $H_{\mathrm{C}}=1435 \mathrm{ppm} \%^{-1} \mathrm{RH}$ and $T_{\mathrm{C}}=2168 \mathrm{ppm} \mathrm{K}^{-1}$.

Figure 16 exhibits the linear dependency of the gauge factor $G_{\mathrm{F}}$ on temperature. The absolute value of the factor increases with increasing temperature. The thermal coefficient of the gauge factor is obtained as $-63.8 \mathrm{ppm} \mathrm{K}^{-1}$. This can be explained by the decrease in the substrate stiffness with increasing temperature. Humidity showed no apparent influence on sensitivity.

The results for a sensor with a similar geometric ratio of the electrodes deposited on a LCP substrate are shown in Fig. 17. 
Table 2. Comparison of power consumption for strain-sensing systems.

\begin{tabular}{lrrr}
\hline Sensor & $\begin{array}{r}\text { PI 45/15 } \\
\text { polyimide }\end{array}$ & $\begin{array}{r}\text { LCP 45/15 LCP } \\
\text { LCP } \\
\text { encapsulated }\end{array}$ & $\begin{array}{r}\text { Differential } \\
\text { measurement } \\
\text { polyimide }\end{array}$ \\
\hline Gauge factor $\mathrm{F}_{\mathrm{F}}$ & -1.4 & -1.3 & 2.1 \\
Capacitance & $47.7 \mathrm{pF}$ & $35.5 \mathrm{pF}$ & $2 \times 5 \mathrm{pF}$ \\
Temperature coefficient & $2170 \mathrm{ppm} \mathrm{K}^{-1}$ & $1960 \mathrm{ppm} \mathrm{K}^{-1}$ & $100 \mathrm{ppm} \mathrm{K}^{-1}$ \\
Humidity coefficient & $1435 \mathrm{ppm} \mathrm{\%}^{-1} \mathrm{RH}$ & $55 \mathrm{ppm} \mathrm{\%}^{-1} \mathrm{RH}$ & $240 \mathrm{ppm} \mathrm{\%}^{-1} \mathrm{RH}$ \\
\hline
\end{tabular}

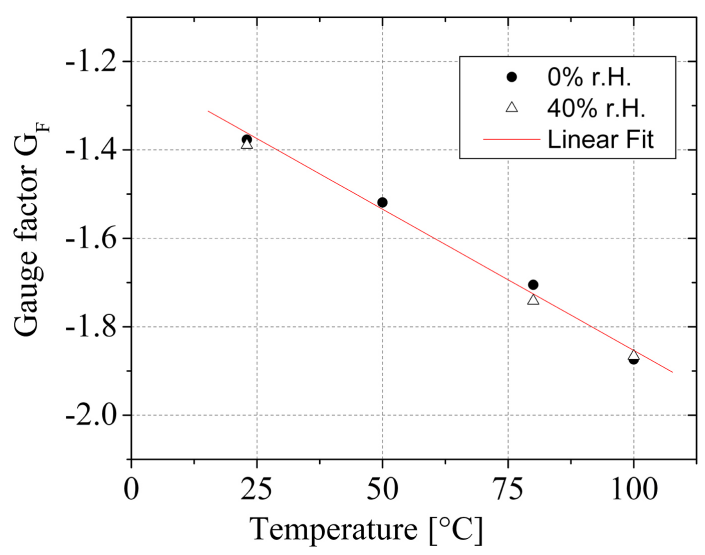

Figure 16. Gauge factor $G_{\mathrm{F}}$ of a single PI-45/15 sensor (design 1) as a function of temperature for relative humidity of 0 or $40 \%$.

For this sensor the measured coefficients are $H_{\mathrm{C}}=1149 \mathrm{ppm} \%^{-1} \mathrm{RH}$ and $T_{\mathrm{C}}=2820 \mathrm{ppm} \mathrm{K}^{-1}$. For an LCP sensor with an encapsulation LCP foil bonded on top, the coefficients $H_{\mathrm{C}}=55 \mathrm{ppm} \%^{-1} \mathrm{RH}$ and $T_{\mathrm{C}}=1958 \mathrm{ppm} \mathrm{K}^{-1}$ are obtained. It is clearly necessary to coat the LCP-based layer with an identical material in order to fully utilize the superior properties of the substrate.

\subsection{Analytical model for capacitive single sensors}

Based on the experimental results for the sensors PI-45/15 and LCP-45/15, we developed a regression model for the capacitance as a function of strain, temperature and relative humidity, which is given in Eq. (9). The coefficients for the sensitivities were obtained from the measured results given in the previous section.

$$
\begin{aligned}
& C(\varepsilon, T, H)=C_{0}\left(1+T_{\mathrm{C}}\left(T-T_{0}\right)+H_{\mathrm{C}}\left(H-H_{0}\right)\right. \\
& \left.+S_{\mathrm{C}} \varepsilon\left(1+T_{\mathrm{C}, \mathrm{SC}}\left(T-T_{0}\right)\right)\right)
\end{aligned}
$$

Coefficients for the sensor PI-45/15 are

$T_{\mathrm{C}}=2168 \mathrm{ppm} \mathrm{K}^{-1} C_{0}=47.74 \mathrm{pF}$,

$H_{\mathrm{C}}=1435 \mathrm{ppm} \%^{-1} \mathrm{RH} T_{0}=23^{\circ} \mathrm{C}$,

$S_{\mathrm{C}}=-13783 \mathrm{ppm} \%^{-1} H_{0}=0 \% \mathrm{RH}$,

$T_{\mathrm{C}, \mathrm{SC}}=-63.8 \mathrm{ppm} \mathrm{K}^{-1}$.

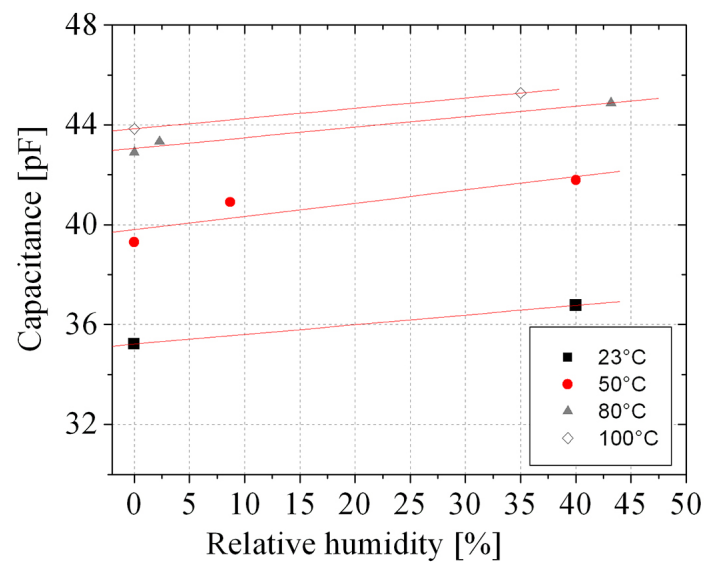

Figure 17. Capacitance of a single LCP-45/15 sensor (design 1) as a function of relative humidity at different temperatures.

Coefficients for the sensor LCP-45/15 are

$T_{\mathrm{C}}=1958 \mathrm{ppm} \mathrm{K}^{-1} C_{0}=35.45 \mathrm{pF}$,

$H_{\mathrm{C}}=55 \mathrm{ppm} \%^{-1} \mathrm{RH} T_{0}=23^{\circ} \mathrm{C}$,

$S_{\mathrm{C}}=-12660 \mathrm{ppm} \%^{-1} H_{0}=0 \% \mathrm{RH}$,

$T_{\mathrm{C}, \mathrm{SC}}=-63.8 \mathrm{ppm} \mathrm{K}^{-1}$ (value of PI).

With these models one can predict the value of the sensor capacitance if the environmental conditions and the level of strain are known. Furthermore, the models provide the strain of a specimen if the sensor capacitance, temperature and humidity are given.

\subsection{Differential measurement with instrumentation IC}

A capacitance-to-digital converter IC (Analog Devices, AD7152) measured the differential capacitances of two PI$45 / 15$ sensors arranged perpendicularly on a flexible circuit as presented in Sect. 4. Figure 18 depicts the linear output signal during the strain measurement.

The gauge factor $G_{\mathrm{F}}$ for this polyimide-based system is +2.1 , the temperature sensitivity is $100 \mathrm{ppm} \mathrm{K}^{-1}$ and the sensitivity against humidity is $240 \mathrm{ppm} \%^{-1} \mathrm{RH}$. The capacitance disparity of the two sensors in this half-bridge is about $2 \%$. This fact explains the low, but not completely compensated for, temperature cross sensitivity of the system. In 


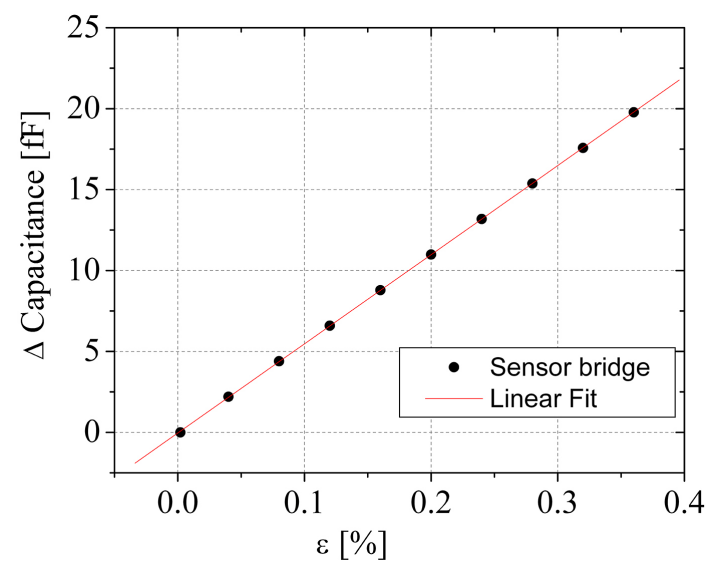

Figure 18. Differential capacitance of two orthogonal PI sensors (design 3) measured with an integrated AD7152 read-out IC as a function of strain at room temperature.

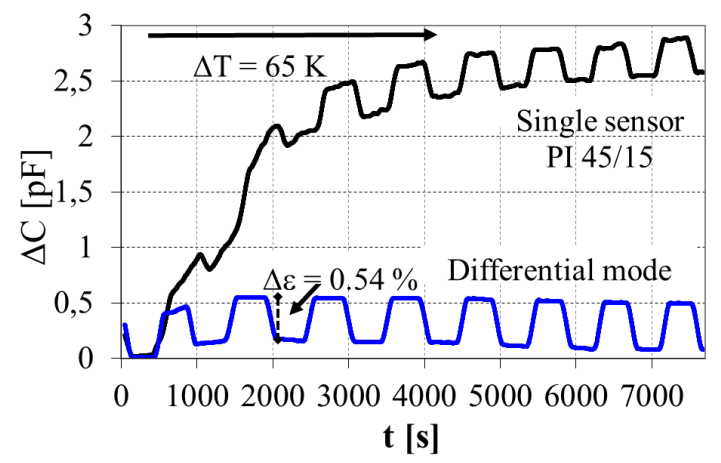

Figure 19. Comparison of the output signal of a single sensor (design 1) and a differential sensor (design 2), both on PI, during a simultaneous variation of the strain $(\Delta \varepsilon=0.54 \%)$ and temperature $(\Delta T=65 \mathrm{~K})$.

Fig. 19, the capacitance of a single sensor is compared with the output signal of the half bridge when the temperature is raised by $65 \mathrm{~K}$ during the measurement. The capacitance of the single sensor increases by $2.5 \mathrm{pF}$, whereas the differential signal increases by only $0.08 \mathrm{pF}$. Hence, the influence of temperature is nearly compensated for. The characterization results are summarized in Table 2 .

\subsection{Power consumption of system for wireless strain measurement}

We developed two demonstrator systems on PCBs for strain measurement, one of which using the novel capacitive gauges and the other with standard resistive sensors $(R=150$ $\Omega$ ) (Joerger, 2010). In these systems, the collected data are stored in a memory-saving mode, and sleep and wake-up routines were implemented to save power. The micro-controller used in these systems already provides a wireless interface
Table 3. Comparison of power consumption for strain-sensing systems.

\begin{tabular}{lrr}
\hline $\begin{array}{l}\text { Power } \\
\text { consumption }\end{array}$ & $\begin{array}{r}\text { System with } \\
\text { commercial resistive } \\
\text { strain gauges }\end{array}$ & $\begin{array}{r}\text { System with } \\
\text { capacitive } \\
\text { strain gauges }\end{array}$ \\
\hline $\begin{array}{l}\text { Electronics } \\
\text { Sensor-element }\end{array}$ & $<1 \mathrm{~mW}$ & $<0.6 \mathrm{~mW}$ \\
\hline Total & $54 \mathrm{~mW}$ & $<0.01 \mathrm{~mW}$ \\
\hline
\end{tabular}

for sending and receiving data via an antenna. The measurement circuits were assembled with low-power devices.

Both systems measured strain of a test specimen and the power consumption was monitored. In Table 3 , the results for the systems are presented.

The power consumption of the instrumentation electronics for both systems was below $1 \mathrm{~mW}$, the application of the low power capacitance to digital converter reduced the power by a factor of 2 . The power consumption of the resistive sensor elements was 40 times higher than for the capacitive sensors.

\section{Summary and conclusions}

The capacitive strain gauges presented in this work were first designed and optimized based on an analytical approach in order to achieve maximum strain sensitivity and a linear output signal. FEM simulations of different sensor configurations verified the analytical model. The analyses of influencing geometry and material parameters led to the establishment of design rules for the strain gauges.

The sensors were fabricated using thin-film technology on two polymer foil substrates by means of a temporary bonding process specifically developed in this work. The characterized capacitive transducers exhibited strain sensitivities that are in the same range as those of standard resistive gauges. The characterization of single capacitive structures revealed high material-dependent cross sensitivities against temperature and humidity. Therefore, a sensor bridge design was introduced that compensates for parasitic effects from differential measurement with a low-power instrumentation IC. The gauge factor of a prototype sensor system was $65 \%$ higher than a single strain gauge on polyimide; the sensitivity for humidity was reduced by $87 \%$ and the influence of temperature by $98 \%$. Furthermore LCP proved to be a superior material when it was used for the substrate and the capping.

The conclusion of this work is that the proposed capacitive strain gauge is a suitable alternative to common resistive strain gauges. The detailed characterization of the sensitivities on strain, temperature and humidity and the linearity of the sensor capacitance on strain are aspects that have not yet systematically been taken into account for capacitive strain gauges (Matsuzaki et al., 2007; Aebersold et al., 2006; Arshak et al., 2000). The sensor structures have significant 


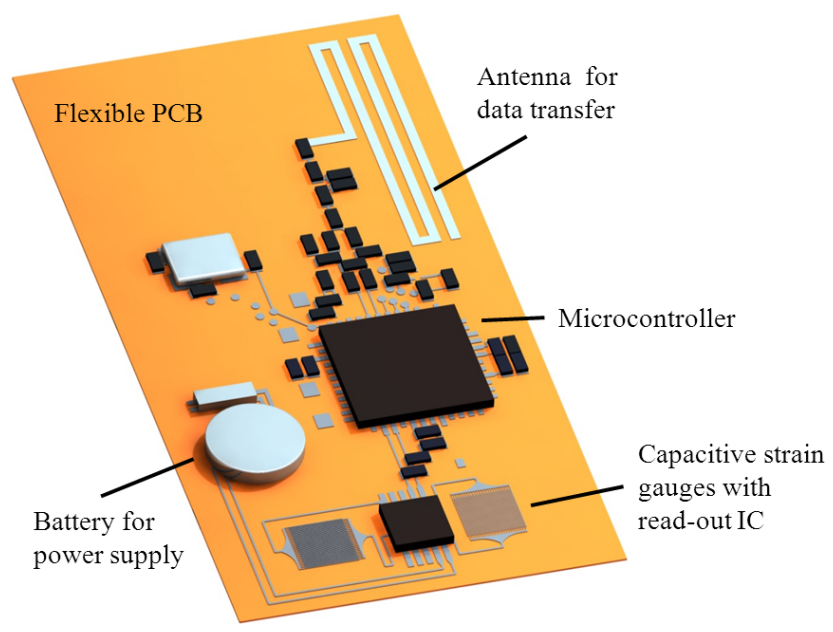

Figure 20. Concept of a wireless, intelligent sensor node with integrated capacitive strain gauges realized on a flexible substrate.

cross sensitivities against temperature and humidity. These can present limiting factors for applications in harsh environments. This work has shown that these factors can be reduced by appropriate dielectric materials. Furthermore, a very effective compensation is possible by differential measurement of orthogonal structures. An integrated system consisting of sensor elements and instrumentation electronics, including a micro-controller for data storage on a flexible substrate, was developed. The power consumption of the systems could be decreased by a factor of 10 by applying capacitive strain gauges instead of standard resistive sensors.

\section{Outlook}

Further steps of development will be the hybrid integration of the capacitive gauges in a wireless, intelligent sensor system realized on a flexible PCB. Figure 20 depicts a scheme of such a system with integrated capacitive strain gauges on a flexible substrate. The future perspective of such a system is a sensor node for detecting of temperature, humidity and strain that is applicable as an energy-harvesting smart label.
Acknowledgements. Part of this work was performed on behalf of the Forschungsvereinigung Antriebstechnik e. V. (FVA) using funding provided by the German Federal Ministry of Economics and Technology (BMWi) pursuant to resolution number $346 \mathrm{ZN}$ passed by the German federal parliament. The authors are grateful for this support.

Edited by: R. Maeda

Reviewed by: two anonymous referees

\section{References}

Aebersold, J., Walsh, K., Crain, M., Voor, M., Martin, M., Hnat, W., Lin, J., Jackson, D., and Naber, J.: Design, modeling, fabrication and testing of a MEMS capacitive bending strain sensor, J. Phys. Conf. Ser., 34, 124-129, 2006.

Arshak, K. I., McDonagh, D., Durcan, and M. A.: Development of new capacitive strain sensors based on thick film polymer and cermet technologies, Sensor. Actuator., 79, 102-114, 2000.

DuPont: Kapton polyimide film - summary of properties, DuPont, 24, p. 5, 2008.

Igreja, R. and Dias, C. J.: Analytical evaluation of the interdigital electrodes capacitance for a multi-layered structure, Sensor. Actuator. A-Phys., 112, 291-301, 2004.

Joerger, T.: Wireless sensor-systems with energy-optimized strain measurement, B.Sc. thesis, Dept. Micro-systems Eng., Univ. of Freiburg, Freiburg, Germany, 2010.

Mamishev, A. V., Sundara-Rajan, K., Yang, F., Du, Y., and Zahn, M.: Interdigital sensors and transducers, Proc. IEEE, 92, 808845, 2004.

Matsuzaki, R. and Todoroki, A.: Wireless flexible capacitive sensor based on ultra-flexible epoxy resin for strain measurement of automobile tires, Sensor. Actuator., 140, 32-42, 2007.

Melcher, J., Deben, Y., and Arlt, G.: Dielectric effects of moisture in polyimide, IEEE T. Electr. Insul., 24, 31-38, 1989.

Rogers Corporation: Data sheet Ultralam 3000 - liquid crystalline polymer circuit material, Rogers Corporation, 6, p. 3, 2008.

Zhang, X.: Development of SOP module technology based on LCP substrate for high frequency electronics applications, Proc. Electronics Systemintegration Technology Conference, 118-125, 2006. 